\title{
Editorial: Roles and Implications of Functional Traits and Phylogenies to Characterize Refugia Under Increasing Climate Variability
}

\author{
Matteo Marcantonio ${ }^{1}$, Fernando A. O. Silveira ${ }^{2}$, Gunnar Keppel ${ }^{3}$, Susan Harrison ${ }^{4}$ and \\ Gianluigi Ottaviani ${ }^{5 *}$ \\ 1 Evolutionary Ecology and Genetics Group, Biodiversity Research Centre, Earth and Life Institute (ELI), UCLouvain, \\ Louvain-la-Neuve, Belgium, ${ }^{2}$ Department of Genetics, Ecology and Evolution, Federal University of Minas Gerais, Belo \\ Horizonte, Brazil, ${ }^{3}$ UniSA STEM and Future Industries Institute, University of South Australia, Adelaide, SA, Australia, \\ ${ }^{4}$ Department of Environmental Science and Policy, University of California, Davis, Davis, CA, United States, ${ }^{5}$ Institute of \\ Botany, The Czech Academy of Sciences, Treboň, Czechia
}

Keywords: biodiversity conservation, eco-evolutionary processes, functional diversity (FD), phylogenetic diversity (PD), trait-based approaches

Editorial on the Research Topic

\section{OPEN ACCESS}

Edited and reviewed by: Peter Convey, British Antarctic Survey (BAS), United Kingdom

*Correspondence:

Gianluigi Ottaviani gianluigi.ottaviani@gmail.com

Specialty section:

This article was submitted to Biogeography and Macroecology,

a section of the journal

Frontiers in Ecology and Evolution

Received: 06 August 2021

Accepted: 31 August 2021

Published: 27 September 2021

Citation:

Marcantonio M, Silveira FAO, Keppel G, Harrison S and Ottaviani G

(2021) Editorial: Roles and Implications of Functional Traits and Phylogenies to Characterize Refugia Under Increasing Climate Variability.

Front. Ecol. Evol. 9:754451. doi: 10.3389/fevo.2021.754451
Roles and Implications of Functional Traits and Phylogenies to Characterize Refugia Under Increasing Climate Variability

\section{REFUGIA: WHAT THEY ARE AND WHY THEY MATTER}

Refugia are locations in which populations and communities are sheltered from adversity, tend to persist during periods of rapid environmental change, and from which they may recolonize surrounding habitats if and when conditions improve. Thanks to their long-term environmental stability, the floras and faunas in refugia are rich in endemic species and unique genotypes; refugia therefore have contributed greatly to the formation of global biodiversity hotspots (Harrison and Noss, 2017; Keppel et al., 2018). In our present era of rapid human-caused environmental changes, refugia have become an ever-increasing research subject for ecologists, biogeographers, and conservation biologists (Keppel et al., 2012; Morelli et al., 2016, 2020).

The refugium concept is flexible, encompassing a diversity of underlying causes (e.g., ability to buffer against aridity, fire or cyclones; Reside et al., 2014), and a broad spectrum of spatial scales (i.e., microrefugia or macrorefugia; Rull, 2009; Keppel et al., 2012). Approaches to identifying and studying refugia are similarly wide ranging, including traditional descriptive and systematic studies and, more recently, testing a priori hypotheses about (inter alia) how refugial processes shape the diversity of functional traits and phylogenetic lineages (Keppel et al., 2018). This Research Topic is motivated by the conviction that functional (trait-based) and phylogenetic approaches can provide insights into the eco-evolutionary dynamics of refugia, including predictions about their future trajectories under increasing climate variability. Integrative research examining functional and phylogenetic patterns in refugia has thus far addressed only a handful of regions, habitats, and/or organisms (e.g., Australian rainforest plants; Kooyman et al., 2011). With this Research Topic, we expand the biogeographic and taxonomic scope of such research. 


\section{FUNCTIONAL AND PHYLOGENETIC APPROACHES TO EXAMINE ECO-EVOLUTIONARY DYNAMICS AND FUNCTIONING OF REFUGIA}

The seven manuscripts in this Research Topic provide a multifaceted perspective on refugia. Surveying the functional traits of ant communities, Bátori et al. illustrated the importance of fine-scale environmental heterogeneity associated with karst depressions providing microrefugia embedded in Central European homogeneous arable landscapes. Refugial communities were characterized by functionally distinct ant assemblages distinguished by functional groups rarely found in the surroundings (e.g., species preferring both warmer, drier and cooler, moister conditions). Similarly, Deák et al. examined the taxonomic, functional, and phylogenetic diversity of plant species on burial mounds (kurgans; up to $\sim 5,000$ yo) scattered in the Eurasian grassland landscape. Kurgans had a taxonomically and functionally diverse community of specialist species compared to surrounding habitats, possibly because of a greater variety of microclimate and soil conditions, but not higher phylogenetic diversity.

Phylogenetic information alone can also provide important insights into the role of refugia. Procheş et al. showed that Southeast Asia hosts ancient lineage diversity, and therefore likely functioned as an important macrorefugium in the past. They discussed the importance of this biodiversity reservoir in the light of a recolonization that will be required at some stage due to the ongoing biodiversity loss. Acevedo and Sandel identified hotspots of phylogenetic endemism for North American birds; high endemism was linked to both shortand long-term climate stability with little impact from landuse changes. This strongly suggests a key role of refugia in maintaining biodiversity.

Huxley and Spasojevic examined how plant communities on alpine tundra sky islands changed over a 12-year interval in relation to climatic change (e.g., increased temperature) and landscape configuration. Species richness and phylogenetic diversity increased over time and with refugial area, while leaf traits' functional diversity decreased shifting toward enhanced stress-tolerance. For the management of refugia, these findings highlight the need to (i) consider temporal, environmental and landscape configuration parameters simultaneously, and (ii) prioritize those patches displaying the highest conservation value and likely capacity to mitigate climate change impacts.

Barthelemy et al. developed an eco-evolutionary model to explore whether taxonomic, functional, and phylogenetic composition of extant species assemblages carries the imprint of past restrictions of refugia. Their simulations suggest that contraction of refugia likely left different signatures on present-day facets of biodiversity. Extant taxonomic and phylogenetic diversity were both maximized by older refugia (i.e., 3,000 generations ago), but the latter metric conveys the signature of past refugia size. The interaction between past refugia expansion, diversification dynamics and trait evolution determined functional diversity, which was higher under the combination of larger refugia that allowed for higher phylogenetic diversification, highlighting the complex eco-evolutionary processes affecting current trait diversity.

While protected areas are not necessarily refugia from an evolutionary perspective, they are increasingly functioning as such because anthropogenic impacts have altered the surrounding landscape to become unsuitable for many species. Protected areas may therefore constitute important biodiversity reservoirs for recolonization-if landscape use keeps changing. In this context, Llorente-Culebras et al. showed that the protected areas of the Iberian Peninsula capture the taxonomic, functional, and phylogenetic diversity of tetrapod assemblages well, emphasizing their potential for future recolonization.

\section{CONCLUSIONS AND PERSPECTIVES FOR REFUGIA-ORIENTED RESEARCH}

The articles in this Research Topic spotlight the multifaceted nature of refugia. They illustrate how different approaches, particularly those integrating functional and phylogenetic aspects, can provide insights into the complex eco-evolutionary dynamics of refugia. Furthermore, all studies unambiguously show the importance of refugia for the conservation, distribution, ecology, and evolution of biodiversity, regardless of the temporal and spatial extent. Refugia were characterized by biotas defined by distinct functional and phylogenetic signatures (compared to surrounding non-refugial landscapes; Keppel et al., 2018), indicating that they are important biodiversity reservoirs and potential sources for natural or assisted recolonization of surrounding landscapes. Seen from this perspective, refugia may act as "insurance policies" for maintaining biological diversity (either genetic, taxonomic or functional) during global changes. A standardized methodology and plan to identify "high-capacity" refugia-those that can buffer against multiple stressors and serve as safe havens for different organism types (Keppel et al., 2015) - may therefore be key to mitigate biodiversity loss, and related deterioration of ecosystem functioning. Applying such an integrated approach would require a global and multifaceted overview of refugia, which would enormously benefit from research on those areas of the world-e.g., Central America, North and Central Africa, Southeast Asia, Arctic and Antarctic regions-that remain largely unknown from a refugial perspective.

\section{AUTHOR CONTRIBUTIONS}

MM and GO wrote the first draft of the Editorial, with substantial inputs from all coauthors during revision. All authors contributed to the article and approved the submitted version. 


\section{FUNDING}

GO was supported by the Czech Academy of Sciences (RVO 67985939). FAOS was supported by FAPEMIG and CNPq. MM acknowledges funding from the Université Catholique de Louvain (UCL), the Fédération Wallonie-Bruxelles (Grant ARC 17/22-086), and the Belgian National Fund for Scientific Research FNRS (Grant PDR FNRS-PDR T.0169.21).

\section{REFERENCES}

Harrison, S., and Noss, R. (2017). Endemism hotspots are linked to stable climatic refugia. Ann. Bot. 119, 207-214. doi: 10.1093/aob/mcw248

Keppel, G., Mokany, K., Wardell-Johnson, G. W., Phillips, B. L., Welbergen, J. A., and Reside, A. (2015). The capacity of refugia for conservation planning under climate change. Front. Ecol. Environ. 13, 106-112. doi: 10.1890/140055

Keppel, G., Ottaviani, G., Harrison, S., Wardell-Johnson, G. W., Marcantonio, M., and Mucina, L. (2018). Towards an eco-evolutionary understanding of endemism hotspots and refugia. Ann. Bot. 122, 927-934. doi: $10.1093 / \mathrm{aob} / \mathrm{mcy} 173$

Keppel, G., Van Niel, K. P., Wardell-Johnson, G. W., Yates, C. J., Byrne, M., Mucina, L., et al. (2012). Refugia: identifying and understanding safe havens for biodiversity under climate change. Glob. Ecol. Biogeogr. 21, 393-404. doi: $10.1111 / j .1466-8238.2011 .00686 . x$

Kooyman, R., Rossetto, M., Cornwell, W. K., and Westoby, M. (2011). Phylogenetic tests of community assembly across regional to continental scales in tropical and subtropical rain forests. Glob. Ecol. Biogeogr. 20, 707-716. doi: 10.1111/j.1466-8238.2010.00641.x

Morelli, T. L., Barrows, C. W., Ramirez,. A. R., Cartwright, J. M., Ackerly, D. D., Eaves, T. D., et al. (2020). Climate-change refugia: biodiversity in the slow lane. Front. Ecol. Environ. 18, 228-234. doi: 10.1002/fee.2189

Morelli, T. L., Daly, C., Dobrowski, S. Z., Dulen, D. M., Ebersole, J. L., Jackson, S. T., et al. (2016). Managing climate change refugia for climate adaptation. PLoS ONE 11:e0159909. doi: 10.1371/journal.pone.0159909

\section{ACKNOWLEDGMENTS}

We thank all the reviewers involved in the revision process for providing prompt comments and thoughtful insights which improved the quality of the research contained in this Research Topic, and the Editor Peter Convey for his suggestions during the revision of this Editorial.

Reside, A. E., Welbergen, J. A., Phillips, B. L., Wardell-Johnson, G. W., Keppel, G., Ferrier, S., et al. (2014), Characteristics of climate change refugia for Australian biodiversity. Aust. Ecol. 39, 887-897. doi: 10.1111/aec.1 2146

Rull, V. (2009). Microrefugia. J. Biogeogr. 36, 481-484. doi: 10.1111/j.1365-2699.2008.02023.x

Conflict of Interest: The authors declare that the research was conducted in the absence of any commercial or financial relationships that could be construed as a potential conflict of interest.

Publisher's Note: All claims expressed in this article are solely those of the authors and do not necessarily represent those of their affiliated organizations, or those of the publisher, the editors and the reviewers. Any product that may be evaluated in this article, or claim that may be made by its manufacturer, is not guaranteed or endorsed by the publisher.

Copyright (C) 2021 Marcantonio, Silveira, Keppel, Harrison and Ottaviani. This is an open-access article distributed under the terms of the Creative Commons Attribution License (CC BY). The use, distribution or reproduction in other forums is permitted, provided the original author(s) and the copyright owner(s) are credited and that the original publication in this journal is cited, in accordance with accepted academic practice. No use, distribution or reproduction is permitted which does not comply with these terms. 